\title{
Highly Nonlinear Silicon Photonics Slot Waveguides without Free Carrier Absorption Related Speed-Limitations
}

T. Vallaitis (1), C. Koos (1), B. Esembeson (2), I. Biaggio (2), T. Michinobu (3),

F. Diederich (3), P. Dumon (4), R. Baets (4), W. Freude (1), J. Leuthold (1)

1 : Institute of High-Frequency and Quantum Electronics, University of Karlsruhe, 76131 Karlsruhe, Germany

2 : Department of Physics, Lehigh University. Bethlehem, PA 18015. USA

3 : Laboratorium für Organische Chemie, ETH Zürich, Honggerberg, $\mathrm{HCl}, \mathrm{CH}-8093$ Zurich, Switzerland

4 : Photonics Research Group, Ghent University-IMEC. B-9000 Gent, Belgium

Email: T.Vallaitis@ihq.uka.de,W.Freude@ihq.uka.de,J.Leuthold@ihq.uka.de

\begin{abstract}
Nonlinear losses of highly nonlinear silicon/organic photonics slot waveguides are analyzed. Unlike silicon strip waveguides, slot waveguides do not show absorption related speed limitations up to highest input powers.
\end{abstract}

\section{Introduction}

Highly nonlinear waveguides are key components for all-optical on-chip processing at highest bit rates. Of particular interest is the Kerr effect as it offers ultimate speed. Unfortunately, the effect is usually very week and therefore on-chip operation is difficult to achieve. Recently, ultrafast operation has been demonstrated taking advantage of record high third order nonlinear effects in ultra-densely confined silicon strip [1] and hybrid silicon/organic slot waveguides [2]. While both designs show highest nonlinearities, the limitations due to free carrier effects is not clear.

In this paper we show that silicon/organic slot waveguides experience negligible losses due to free carriers generated by two-photon absorption. This enables operation up to highest speed, not limited by free carrier lifetime limitations. Strip waveguides fabricated on the same chip show long lasting FCA effects that might ultimately limit operation.

\section{Waveguide Structure and Experimental Setup}

The structure of the highly nonlinear silicon-oninsulator (SOI) strip and slot waveguides are shown as insets of Figure 1. They comprise of silicon (Si) ribs on a silica $\left(\mathrm{SiO}_{2}\right)$ buffer. The waveguides are filled and covered with a Kerr-type nonlinear (NL) organic cladding (DDMEBT) [3] with a refractive index of $n=1.8$. The nonlinearity of the slot waveguides is further enhanced due to the discontinuity of the normal electric field at the interface, up to a record value of $\gamma=10^{5} \mathrm{~W}^{-1} \mathrm{~km}^{-1}$ [2]. Waveguide dimensions and coupling losses are given in Table 1.

We used a heterodyne pump-probe technique with sub-picosecond resolution to measure transmission amplitude and phase dynamics [4]. Pulses with a FWHM of $100 \mathrm{fs}$ and a center wavelength of $1550 \mathrm{~nm}$ are generated by an optical parametric oscillator with a repetition rate of $80.42 \mathrm{MHz}$. Pump, probe and reference pulses are co-polarized (TE). After passing the waveguide, a Michelson interferometer with

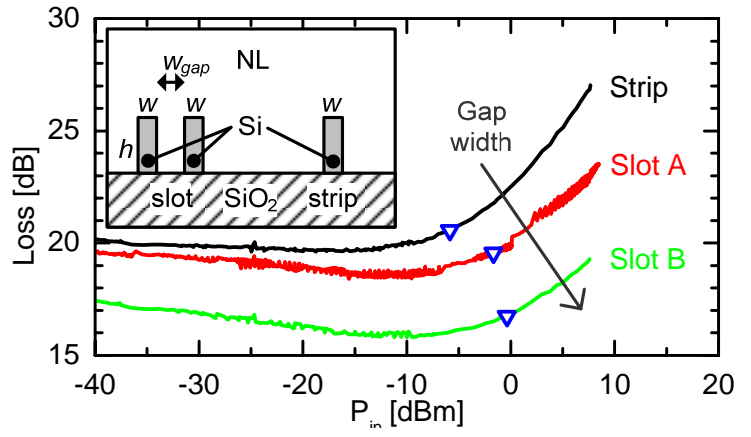

Figure 1: Insertion loss of the waveguide including coupling losses to lensed fibers for strip (black) and slot waveguides (red/green) performed with 100 fs pulses at $\lambda=1550 \mathrm{~nm}$. Strip waveguides have higher nonlinear losses that set in at lower power levels

unbalanced arm lengths is used to superimpose probe and reference pulses such that a lock-in amplifier can detect amplitude $T$ and phase $\varphi$ of the transmitted signal. With the effective length $L$ and the wavelength $\lambda$, the change of refractive index can be calculated from $\Delta n=-\Delta \varphi \lambda /(2 \pi L)$, (time dependency $\exp (j \omega t))$. To investigate insertion losses, probe and reference paths are blocked and the average power of input and output pulses is measured in the fiber.

\begin{tabular}{|l|l|l|l|}
\cline { 2 - 4 } \multicolumn{1}{c|}{} & Strip & Slot A & Slot B \\
\hline \hline Length $L[\mathrm{~mm}]$ & 4 & 4 & 4 \\
\hline Height $h[\mathrm{~nm}]$ & 220 & 220 & 220 \\
\hline Width $w[\mathrm{~nm}]$ & 375 & $2 \times 216$ & $2 \times 212$ \\
\hline Gap $w_{\text {gap }}[\mathrm{nm}]$ & - & 157 & 205 \\
\hline$\alpha[\mathrm{dB} / \mathrm{mm}]$ & $0.8^{*}$ & 1.55 & 1.48 \\
\hline$a_{\mathrm{cp}}[\mathrm{dB}]$ & $8.2^{*}$ & 6.2 & 4.1 \\
\hline
\end{tabular}

Table 1: Waveguide dimensions and parameters. The quantity $a_{\mathrm{cp}}$ denotes the fiber-chip coupling loss per facet in $d B$. The values denoted with an asterisk $\left(^{*}\right)$ were obtained from waveguides with a $\mathrm{SiO}_{2}$ cover. 

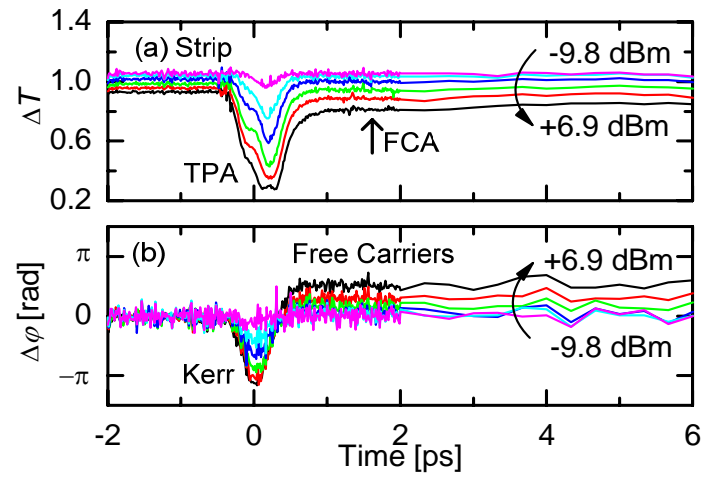

Figure 2: (a) Change of transmission $\Delta T$ and (b) phase in a strip waveguide with pronounced Kerr and FCA nonlinearities for different input powers. The recovery time of the device is limited by the FCA.

\section{Experimental Results}

Figure 1 shows the input power dependence of the insertion loss. Two effects can be seen. At low input powers we find a saturable absorption in the polymer. This effect is extremely slow and will average out above $100 \mathrm{Mbit} / \mathrm{s}$. Losses further decrease with gap width as the light guided in the polymer intensifies. Figure 1 further shows that at higher input powers strip waveguides exhibit higher nonlinear losses. This effect is due to the TPA and related FCA, with lifetimes that could pose a challenge for communications and need further investigation.

The dynamics of the various nonlinear effects in the strip and slot waveguides have been derived by pump-probe measurment depicted in Figs. 2 and 3. for input powers between $-9.8 \mathrm{dBm}$ and $+6.9 \mathrm{dBm}$. The transmission of the strip waveguide in Fig. 2(a) shows the two photon absorption (TPA) at $t=0$ and absorption by free carriers (FCA) with nanosecond lifetimes. The phase evolution (b) shows the Kerreffect at $t=0$, followed by the FCA phase change [5].

Results of the pump-probe measurements of the slot waveguide are shown in Fig. 3. The transmission (a) shows weak two-photon absorption and a strong spectral artifact. Most remarkably, there is no measurable free carrier absorption. This is supported

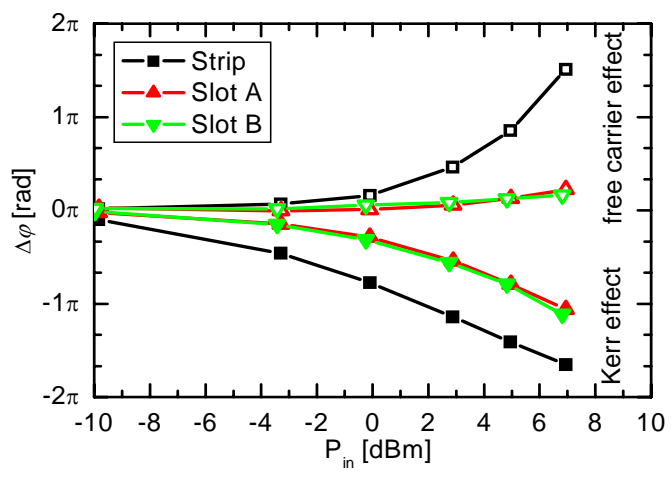

Figure 4: Phase change due to Kerr (solid symbols) and free carrier effect (open symbols) for strip and slot waveguides. In strip waveguides the free carriers dominate at high input powers.

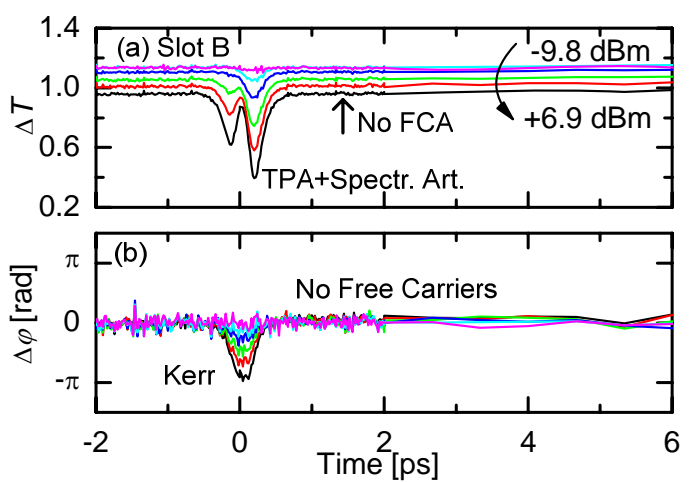

Figure 3: (a) Change of transmission $\Delta T$ and (b) phase in a slot waveguide for different input powers with pronounced Kerr nonlinearities. A free carrier limitation is not observed.

by the phase measurement in Fig. 3(b) that shows a strong Kerr-effect but no measurable free carrier effect. As a consequence the device's recovery follows the input pulse width. The spectral artifact at $t=0$, is due to the strong frequency shift which leads to artlfacts in our heterodyne detection but has no impact on the measured phase [6].

Figure 4 compares the phase changes of Kerr and free carrier effect of Figs. 2 and 3 . In strip waveguides, a stronger Kerr nonlinearity is partly compensated by the free carrier effect with opposite sign. In slot waveguides the Kerr effect though slightly weaker, ultimately dominates.

\section{Conclusions}

Hybrid silicon/organic waveguides in slot waveguide geometry exhibit high nonlinearities and low losses. The generation of free carriers due to two-photon absorption is greatly reduced compared to strip waveguides. This will allow higher power levels and avoid patterning effects.

This work was supported by the Center for Functional Nanostructures (CFN) of the Deutsche Forschungsgemeinschaft (DFG), by the Initiative of Excellence of the University of Karlsruhe and by the European project TRIUMPH (grant IST-027638 STP). FD and TM acknowledge support from the ETH research council. IB and BE acknowledge partial support from the Commonwealth of Pennsylvania, Ben Franklin Technology Development Authority. Fabrication was done through the ePIXnet silicon photonics platform.

\section{References}

1. Salem et al., Nature Photonics 2, 35 (2008)

2. Koos et al., Proc. Opt. Fiber Communication Conf. (OFC'08), PDP25 (2008)

3. Michinobu et al., Chem. Commun. 737 (2005)

4. Vallaitis et al., Opt. Express, 16, 170 (2008)

5. Dekker et al., Opt. Express. 14, 8336 (2006)

6. Mørk et al., J. Opt. Soc. Am. B, 13, 1803 (1996) 